\title{
Zu Dr. C. Parrot's „Beiträge zur Ornithologie Sumatras und der Insel Banka".
}

\author{
Von Max Bartels, Pasir Datar (Java).
}

In den Abhandlungen der Königlich Bayerischen Akademie der Wissenschaften Band XXIV erschien im Jahre 1907 aus der Feder des bekannten Ornithologen Dr. C. Parrot-Mürıchen, eine sehr interessante Arbeit über die Ornithologie Sumatras und der Insel Banka. - Da in dieser Arbeit auch eine ziemlich grofse Anzahl javanischer Vögel besprochen wird, sei es mir gestattet, an dieser Stelle, auf Grund meiner langjährigen Beobachtungen der Vogelwelt Javas, zu den Ausführungen Dr. Parrot's einige Bemerkungen $\mathrm{zu}$ veröffentlichen.

Microhierax tringillarius, Drap. Sharpe, C. B. B. M. $\left.{ }^{1}\right)$ I. p. 367. Dr. C. Parrot. A. K. B. A. W. ${ }^{2}$ ) Microhierax coerulesctens fringillarus Drap. p. 157.

Die Verschiedenheit des rostbraunen Tones der Unterseite ist auch mir stets aufgefallen. Ich halte die Stücke, bei denen derselbe am stärksten ausgeprägt ist, für sehr alt. Die bedeutenden Gröfsenunterschiede beruhen auf Geschlechtsdifferenz.

\section{Spizaetus limnaetus Horsf. - Spizaetus caligatus, Raffl.} Sharpe. C. B. B. M. I. p. 272.

Dr. C. Parrot. A. K. B. A. W. Spizaetus cirrhatus limnaetus, Horsf. et Spizaetus cirrhatus caligatus, Raffl. pp. 159/160.

Obwohl ich die Frage der artlichen Trennung von Spizaetus limnaetus Horsf. und Spizaetus caligatus Raffl. vorläufig noch unentschieden lassen möchte, kann ich doch mit Bestimmtheit behaupten, dafs die dunkle Phase kein Alterskleid der hellen darstellt, wie auch Herr Dr. Parrot richtig hervorhebt, denn es befinden sich in unserer Sammlung alte und jungedunkle Exemplare. ${ }^{3}$ )

Einen Schopf besitzen weder die dunklen noch die hellen Vögel. In Java werden von den Eingeborenen keine derartigen Federn als Kopfschmuck getragen und kann also von einem Ausreifsen der Schopffedern zu diesem Zwecke keine Rede sein, auch

1) Catalogue of Birds in the collection of the British Museum.

2) Abhandlungen der Königlich Bayerischen Akademie der Wissenschaften XXIV. Bd.

$\left.{ }^{3}\right)$ Nach Dr. Bernstein in „Journal für Ornith. VIII. 1860, p. 419/423“ soll die Farbe der Iris beider Phasen dunkelbraun sein. Dies ist jedoch nicht zutreffend, denn es ist die Iris der sehr alten Exemplare dunkel goldig bis chromgelb gefärbt, letzteres beobachtete ich bisher jedoch nur an alten Männchen der hellen Phase. Eine genaue Beschreibung der verschiedenen Kleider der hellen Phase behalte ich mir für spăter vor, nur möchte ich hier kurz erwähnen, dafs sich meine bisher darüber gemachten Beobachtungen nicht mit denen Dr. Bernsteins decken. 
werden Haubenadler nur sehr selten von Eingeborenen erbeutet. In unserer Sammlung befinden sich augenblicklich 29 zum gröfsten Teil von mir persönlich erlegte, helle und dunkle javanische Haubenadler, von denen keiner mit einem Schopfe geschmückt ist. Nach meiner Ansicht ist es nicht unmöglich, dafs der gehäubte Spizaetus im Leidener Museum irgend einer anderen Art angehört und kein Spizaetus limnaetus ist. Dafs Haubenadler anderer Länder nach Java verfliegen, wurde durch das von mir gesammelte Männchen von dem ebenfalls gehäubten Spizaetus Kelaarti Legge zur Genüge bewiesen. ${ }^{1}$ )

Hemicercus concretus Temm. Hargitt. C. B. B. M. XVIII. p. 482. Dr. C. Parrot. A. K. B. A. W. Hemicercus concretus sordidus, Eyton p. 180.

Bei sämtlichen alten Männchen unserer Sammlung ist der ganze Oberkopf lebhaft rot gefärbt, der der alten Weibchen dagegen einfach grau. Bei einem jungen Weibchen ist der Vorderkopf auffälligerweise gelbbraun und der Schopf rot gefärbt. Die Geschlechtsangabe „Weibchen" des im Münchner Museum befindlichen jungen Exemplares, von dem Dr. Parrot sagt, dafs es nur ein junges Männchen sein könnte, ist also jedenfalls richtig.

Zanclostomus javanicus Horsf. Shelley. C. B. B. M. XIX. p. 380. Dr. C. Parrot. A. K. B. A. W. Zanglostomus javanicus Horsf. p. 183.

Wie Dr. Parrot richtig annimmt, sind die weifsen Endspitzen der Stofsfedern dieses Kuckucks kein Jugendmerkmal. Bei allen von mir bisher auf Java gesammelten jungen und alten Vögeln dieser Art sind die Endspitzen der Stofsfedern weifs.

Rhamphococcyx curvirostris singularis Parrot.

Dr. C. Parrot. A. K. B. A. W. p. 186.

Nach meiner Ansicht ist dies keine neue „Form", sondern wahrscheinlich ein junger Vogel von Urococcyx erythrognatus Hartl. Shelley. C. B. B. M. XIX p. 398, denn auch bei Jungen des sehr nahe verwandten Khinococcyx curvirostris, Shaw. ${ }^{2}$ ) von Java, ist das zentrale Sto[sfederpaar vollständig grün. Im übrigen sind jüngere Vögel von Rh. curvirostris ebenso wie die Alten gefärbt, haben aber ein sicheres Zeichen, an dem sie sofort von diesen zu unterscheiden sind. Die Stofsfedern sind nämlich schmaler und spitzer auslaufend, wie die der Alten. Sogar die kaum dem Nest entschlüpften Jungen von Rhinococcyx cur-

1) Dr. 0. Finsch. Ein neuer Irrgast für Java (Spizaetus Kelaarti, Legge) "Ornithologische Monatsberichte 1908 pag. 44".

$\left.{ }^{2}\right)$ Auch die von Dr. Parrot in einer Fulsnote erwăhnte merkwürdige Geschlechtsdifferenz in der Irisfärbung ist Khinococcyx curvirostris eigen, denn auch bei ihm ist die Iris des alten Männchens blăulichweifs und die des alten Weibchens gelb gefarrbt.

Joum. f. Orn, LVW, Jahrg. Jali 1910. 
virostris tragen, mit Ausnahme des gänzlich grünen Schnabels und des grünen zentralen Stofsfederpaares, kein von dem der Alten auffallend verschiedenes Kleid, nur einige Handdecken zeigen rostbraune Endsäume, die jedoch so schmal sind, dafs sie sich gewifs schon nach kurzer Zeit durch Abstofsen des Gefieders verlieren. Besonderen Wert scheint Herr Dr. Parrot auf die Schnabelfärbung zu legen, trotzdem diese doch eigentlich beim Vergleichen von Bälgen niemals berücksichtigt werden sollte, da sie sich bekanntlich bei vielen Vögeln bereits kurz nach dem Tode so verändert, dafs von der eigentlichen Färbung nichts mehr übrig bleibt. Der Behauptung Dr. Parrots, wenn es sich bei seinem Vogel um ein Jugendstadium handelte, so mülste dies viel häufiger aufgefunden sein, kann ich entgegenstellen, dafs es auf Java, wo jedenfalls dieselben Verhältnisse herrschen werden, wie auf dem benachbarten Sumatra, unendlich viel leichter ist alte, wie junge Vögel zu sammeln; so befindet sich in unserer Sammlung z. B. unter einer ziemlich bedeutenden Anzahl Rhinococcyx curvirostris nur ein einziges ganz junges Exemplar und dies ist mit vielen anderen Vögeln ebenso der Fall, trotzdem ich mir stets die gröfste Mühe gebe, junge Vögel $\mathrm{zu}$ erbalten. Die oftmals fast undurchdringlichen Dickichte der hiesigen Wälder bieten den jungen Vögeln so vorzügliche Schlupfwinkel, dafs die Erbeutung derselben, namentlich der kaum flügge gewordenen, wirklich ungemein schwierig ist.

Coccystes coromandus L. Shelley. C. B. B. M. XIX. p. 214. Dr. C. Parrot A. K. B. A. W. Coccystes coromandus L. p. 190. Die auffallende Konstanz in der äufseren Erscheinung dieser Art dürfte vielleicht darauf zurückzuführen sein, dafs das Brutgebiet dieses schönen Kuckucks sehr viel kleiner ist wie das Gebiet, in dem er gesammelt wurde. Auf Java wenigstens ist er nach meinen bisherigen Beobachtungen nur Wintergast. Was die scheinbare Seltenheit betrifft, so dürfte diese zum Teil in der sehr versteckten Lebensweise zu suchen sein und weil die schönen Vögel im Winterquartier niemals ihre Stimme ertönen lassen, wodurch ihr Auffinden natürlich ungemein erschwert wird. Dr. Vordermann, der bekanntlich viele Jahre in der Umgegend von Batavia sammeln liefs, bekam hier niemals einen dieser Kuckucke und doch überwintern dieselben ganz in der Nähe des Weichbildes der alten Stadt Batavia, wie ich in den Jahren 1906 und 1907 feststellen konnte.

Centropus nigrorufus Cuv. Dr. O. Finsch. N. f. the L. M. ${ }^{1}$ ) XXIII. 1902 . p. 156.

Centropus purpureus Shelley. C. B. B. M. XIX. p. 348. Dr. C. Parrot. A. K. B. A. W. Centropus sinensis eurycercus Hay p. 108.

1) Notes from the Leyden Museum. 
Herr Dr. Parrot vermag nicht zu glauben, dafs dieser Sporenkuckuck wirklich eine ,very distinct species" ist, wie es im C. B. B. M. heifst und doch ist dies der Fall. Centropus nigrorufus unterscheidet sich von Centropus sinensis nicht nur durch die Färbung und Grölse, sondern auch durch die Wahl seines Aufenthaltsortes, denn ich traf ihn bisher nur im wasserund sumpfreichen 'Tieflande, während Centropus sinensis, in Westjava wenigstens, auch überall an geeigneten Örtlichkeiten im Gebirge vorkommt. Übrigens verweise ich nach der sehr ausführlichen Abhandlung, die von Herrn Professor Dr. O. Finsch in den Notes from the Leyden Museum über diesen Sporenkuckuck veröffentlicht wurde.

Halcyon chloris Bodd. Sharpe. C. B. B. M. XVII. p. 273. Dr. C. Parrot. A. K. B. A. W. Halcyon chloris Bodd. p. 203.

Die Beobachtung von Herrn Dr. Hagen, dafs dieser Eisvogel an die Meeresküste, beziehungsweise an die Brackwasserregion gebunden ist, trifft für Java nicht $z u$, denn man findet ihn an geeigneten Orten, manchmal sogar ziemlich weit vom Wasser entfernt, auch überall im Binnenlande, sogar hoch im Gebirge.

Nach meiner Ansicht liegt hier vielleicht eine Verwechslung mit dem sehr ähnlichen Halcyon sanctus Vig. \& Horsf. vor, den man nämlich auf Java tatsächlich fast nur in den von Herrn Dr. Hagen genannten Regionen antrifft.

Eurylaemus javanicus Horsf. Sclater C. B. B. M. XIV. p. 463. Dr. C. Parrot. A. K. B. A. W. Eurylaemus javanicus Horsf. p. 217.

Wie Herr Dr. Parrot richtig bemerkt, sind die Exemplare mit sehr deutlichem Pectoralband zweifellos Männchen, mir wenigstens ist bisher noch kein Weibchen mit einem solchen Bande zu Gesicht gekommen.

In der Schnabelfärbung scheinen sich die Sumatraner von den Javanern $\mathrm{zu}$ unterscheiden, denn der Schnabel der letztgenannten ist nicht einfarbig dunkel kobaltblau, sondern wie folgt gefärbt: Oberschnabel: Von der Wurzel bis zur Mitte dunkel kobaltblau, vordere Schnabelhälfte deckgrün, nach der Spitze zu in gelblich übergehend, diese, das Nasenloch und ein sich kurz vor dem Mundwinkel verlierender Rand schwarz. Unterschnabel: Kobaltblau, grünlich angehaucht, der durch den Oberschnabel verdeckte Teil des Mundwinkels gelb, daran anschliefsend ein sich nach der Spitze zu verbreiternder schwarzer Rand. Spitze schwarz. Ganz abweichend hiervon präsentiert sich der Schnabel der kaum flügge gewordenen Jungen denn dieser ist sehr einfach dunkelhornbraun gefärbt mit etwas hellerer Spitze.

Ianius tigrinus Drap. Gadow. C. B. B. M. VIII. p. 289. Dr. C. Parrot. A. K. B. A. W. Lanius tigrinus, Drap. p. 232. Dieser Würger ist nach meinen bisherigen Beobachtungen auf Java nur Wintergast. 
Aethopyga siparaja Raffl. Gadow. C. B. B. M. IX. p. 21. Dr. C. Parrot. A. K. B. A. W. Aethopyga siparaja siparaja Raffl. p. 234.

Die Ansicht Dr. Parrot's, dafs Aethopyga mystacalis Temm. die Javaform von Aethopyga siparaja Raffl. ist, kann ich nicht teilen, denn auf Java kommt neben mystacalis auch die echte siparaja Raffl. vor. Zwar ist letztere Art viel weniger verbreitet, wo sie jedoch vorkommt, findet sich auch die viel häufigere Aethopyga mystacalis und leben diese beiden Honigsauger also als gut getrennte Arten nebeneinander.

Die weniger starke Stufung des Stofses von Aethopyga siparaja Raffl. ist kein Zufall, sondern ein konstanter Unterschied zwischen dieser Art und Aethopyga mystacalis Temm.

Prionochilus percussus Temm. Sharpe. C. B. B. M. X. p. 65. Dr. C. Parrot. A. K. B. A. W. Prionochilus percussus ignicapillus Eyton p. 237.

Herr Dr. Parrot bemerkt: „Die Javaform soll völlig schwarzen Schnabel aufweisen." Dies ist nicht richtig, denn der Schnabel des hiesigen Vogels ist zweifarbig. Männchen, Oberschnabel: schwarz, Unterschnabel von der Spitze bis zur Mitte schwarz, Wurzel bleigrau.

Orthotomus cineraceus Blyth. Sharpe. C. B. B. M. VII. p. 225. Dr. C. Parrot. A. K. B. W. Orthotomus cineraceus Blyth. p. 252. Die Bezeichnung „Pritchak" ist, wie Herr Dr. Parrot richtig annimmt, ein Lautname.

\section{Munia maja L. Sharpe. C. B. B. M. XIII. p. 332.}

Dr. C. Parrot. A. K. B. A. W. Munia maya maya L. p. 254.

Die Annahme Dr. Parrot's dafs Munia maja auf Java durch Munia ferruginosa Sparrm. vertreten wird, beruht zweifellos auf einem Irrtum denn hier. ist nach meinen bisherigen Beobachtungeu Munia maja L. weit häufiger, als terruginosa Sparrm. Letztere Art habe ich z. B. noch niemals im Gebirge angetroffen, während Munia maja in den an unsere Plantagen grenzenden Reisfeldern eine sehr häufige Erscheinung ist und auch gern im hohen Grase in der Nähe des Urwaldes nistet. 


\section{$2 \mathrm{BHL}$ Biodiversity Heritage Library}

1910. "Zu Dr. C. Parrot's QBeiträge zur Ornithologie Sumatras und der Insel BankaD.Journal fu

r Ornithologie 58, 484-488. https://doi.org/10.1007/bf02090105.

View This Item Online: $\underline{\text { https://www.biodiversitylibrary.org/item/43496 }}$

DOI: https://doi.org/10.1007/bf02090105

Permalink: https://www.biodiversitylibrary.org/partpdf/142708

\section{Holding Institution}

MBLWHOI Library

\section{Sponsored by}

MBLWHOI Library

\section{Copyright \& Reuse}

Copyright Status: No known copyright restrictions as determined by scanning institution.

This document was created from content at the Biodiversity Heritage Library, the world's largest open access digital library for biodiversity literature and archives. Visit BHL at https://www.biodiversitylibrary.org. 